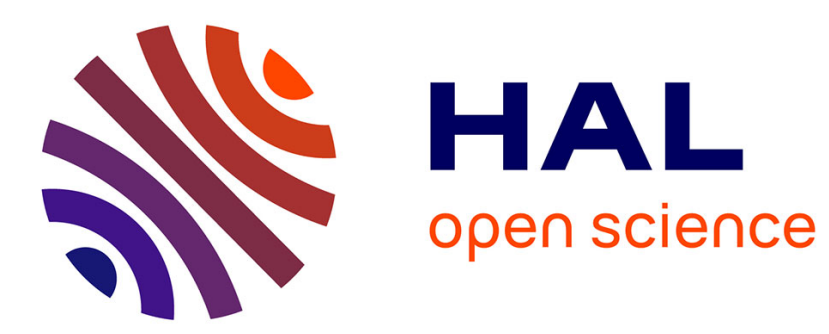

\title{
Robust Output Stabilization of Time-Varying Input Delay Systems using Attractive Ellipsoid Method
} Andrey Polyakov, Alexander Poznyak, Jean-Pierre Richard

\section{To cite this version:}

Andrey Polyakov, Alexander Poznyak, Jean-Pierre Richard. Robust Output Stabilization of TimeVarying Input Delay Systems using Attractive Ellipsoid Method. IEEE Conference on Decision and Control, IEEE, Dec 2013, Florence, Italy. pp.934-939. hal-00920047

\section{HAL Id: hal-00920047 https://hal.inria.fr/hal-00920047}

Submitted on 17 Dec 2013

HAL is a multi-disciplinary open access archive for the deposit and dissemination of scientific research documents, whether they are published or not. The documents may come from teaching and research institutions in France or abroad, or from public or private research centers.
L'archive ouverte pluridisciplinaire HAL, est destinée au dépôt et à la diffusion de documents scientifiques de niveau recherche, publiés ou non, émanant des établissements d'enseignement et de recherche français ou étrangers, des laboratoires publics ou privés. 


\title{
Robust Output Stabilization of Time-Varying Input Delay Systems using Attractive Ellipsoid Method
}

\author{
Andrey Polyakov, Alexander Poznyak, Jean-Pierre Richard
}

\begin{abstract}
The problem of output control design for linear system with unknown and time-varying input delay, bounded exogenous disturbances and bounded deterministic measurement noises is considered. The prediction technique is combined with Luenberger-like observer design in order to provide the stabilizing output feedback. The scheme of parameters tuning for reduction of measurement noises effect and exogenous disturbances effects is developed basing on Attractive Ellipsoids Method. Under some restrictions it is formalized as semidefinite programming problem. The theoretical results are supported by numerical simulations.
\end{abstract}

\section{INTRODUCTION}

Models with time delays frequently appear in networked systems [1], chemical [2] and biological processes [3], automobile [4] and aerospace industries [5]. The real-life applications need control algorithms, which are robust with respect to exogenous disturbances, system uncertainties and measurement noises. The control problems for systems with known delays are studied for both linear and nonlinear systems, state, input and output delays ( see, for example, [6], [7], [8], [9], [10], [11], [12] and references within). However, an important performance index of control systems is a robustness with respect to unknown and time-varying delay. Such analysis for full state feedback control algorithms has been done for systems with linear (see, for example, [13]) and relay feedbacks [14]. In [15], [16] the first order sliding mode control systems with input delay was studied. There are few researches devoted to the output control design for systems with time-varying and unknown delay [17], [18], [19]. However, they study robustness properties of time-delay control systems only with respect to uncertainties in timedelay. Moreover, the adaptive control scheme presented in [17] is applicable only to a chain of integrators.

This paper treats the problem of output control design for linear system with unknown and time-varying input delay,

Andrey Polyakov and Jean-Pierre Richard are with Non-A INRIA LNE, Parc Scientifique de la Haute Borne 40, avenue Halley Bat.A, Park Plaza 59650 Villeneuve d'Ascq(e-mail: andrey.polyakov@inria.fr, jeanpierre.richard@inria.fr)

Alexander Poznyak is with Department of Automatic Control, CINVESTAV, Av. IPN-2508, Col. San Pedro Zacatenco, D.F., Mexico (email:apoznyak@ctrl.cinvestav.mx)

Andrey Polyakov is also with Institute of Control Sciences, Russian Academy of Sciences, Profsouyznaya 65, Moscow, Russia.

Jean-Pierre Richard is also with LAGIS UMR CNRS 8219 and Ecole Centrale de Lille, BP 48, Cité Scientifique, 59651 Villeneuve-d'Ascq, France.

This work was supported by EU Interreg IV A 2 Mers Seas Zeeën Cross-border Cooperation Programme under SYSIASS project 06-020. It was also supported by Ministry of Higher Education and Research, NordPas de Calais Regional Council and FEDER through the Contrat de Projets Etat Region (CPER) CIA 2007-2013. bounded exogenous disturbances and bounded deterministic measurement noises. Two approaches can be considered in order to tackle this problem. The first one is based on finite-time (or fixed-time) observer design for the system with unknown control input [20], [21]. Theoretically such an observer guarantees that after finite (or fixed) period of time the observed states will coincide with the real ones. This property ensures fulfilling of separation principle, so any existing full-state control can be applied. Unfortunately, in practice the exact convergence of the observer cannot be guaranteed due to noises, inaccuracy of digital realization of the continuous-time observer, etc. So, in fact the additional robustness analysis of the closed-loop system is required. This second step does not assume fulfilling separation principle. The stability and robustness analysis in this case has to be done for whole closed-loop system including observer and controller parts. In particular, an output-based control design was presented for delay-free linear [22] and nonlinear (quasiLipschitz) [23] systems using attractive (invariant) ellipsoids method (AEM) [24], [25]. The present paper extends this technique to systems with unknown and time-varying input delay.

The paper is organized as follows. The next section considers notations used in the paper. Section III presents system description and problem statement. Then, the attractive ellipsoid method is introduced and discussed for timedelay systems. Section V.A develops the control algorithm for quasi-Lipschitz nonlinear systems with unknown and time-varying input delay. The scheme for tuning of the control parameters in order to minimize the stabilization error according AEM is introduced. Finally, numerical simulation results are presented.

\section{NOTATIONS}

- $\mathbb{R}$ is a set of real numbers; $\mathbb{R}_{+}:=\{x \in \mathbb{R}: x>0\}$.

- The notation $\operatorname{diag}\left\{\lambda_{1}, \ldots, \lambda_{n}\right\}$ means diagonal matrix with elements $\lambda_{i}$, where $\lambda_{i}$ may be both numbers and square matrices.

- $I_{n}=\operatorname{diag}\{1,1, \ldots, 1\} \in \mathbb{R}^{n \times n}$ is an identity matrix;

- $\lambda_{\max }(P)$ and $\lambda_{\min }(P)$ denote maximum and minimum eigenvalues of the symmetric matrix $P \in \mathbb{R}^{n \times n}$.

- The notation $P>0(<0, \geq 0, \leq 0)$ for $P \in \mathbb{R}^{n \times n}$ means that $P$ is symmetric and positive (negative) definite (semidefinite).

- trace $P$ is a sum of diagonal elements of the matrix $P \in \mathbb{R}^{n \times n}$. 


\section{PROBlem STATEMENT}

Consider the input delay control system of the form

$$
\dot{x}=A x+B u(t-h(t))+D f(t, x), \quad y=C x+E g(t),
$$

where $x \in \mathbb{R}^{n}$ is the system state, $u \in \mathbb{R}^{m}$ is the vector of control inputs, $y \in \mathbb{R}^{k}$ is the measured output, $A \in \mathbb{R}^{n \times n}$, $B \in \mathbb{R}^{n \times m}, C \in \mathbb{R}^{k \times n}, D \in \mathbb{R}^{n \times r}$ and $E \in \mathbb{R}^{k \times p}$ are known matrices. The input delay $h(t)$ is assumed to be unknown but bounded by

$$
0 \leq \underline{h} \leq h(t) \leq \bar{h},
$$

where $h: \mathbb{R} \rightarrow \mathbb{R}$ is a locally measurable function and the numbers $\underline{h}, \bar{h}$ are given. Denote $\Delta h=\bar{h}-\underline{h}$.

The locally measurable function $f: \mathbb{R}^{n+1} \rightarrow \mathbb{R}^{r}$ describes bounded exogenous disturbances and system uncertainties

$$
f^{T}(t, x) Q_{f} f(t, x) \leq 1, \quad \forall t \in \mathbb{R} \text { and } \forall x \in \mathbb{R}^{n},
$$

where $Q_{f} \in \mathbb{R}^{r \times r}$ is a given positive definite matrix.

The locally measurable function $g: \mathbb{R} \rightarrow \mathbb{R}^{p}$ describes bounded deterministic measurement noises

$$
g^{T}(t) Q_{g} g(t) \leq 1, \quad \forall t \in \mathbb{R}
$$

where $Q_{g} \in \mathbb{R}^{p \times p}$ is a given positive definite matrix.

The system (1) is studied with the initial conditions:

$$
\begin{gathered}
x(0)=x_{0} \\
u(t)=v(t) \text { for } t \in[-\bar{h}, 0),
\end{gathered}
$$

where $v: \mathbb{R} \rightarrow \mathbb{R}^{n}$ is some continuous function.

Assumption 1: The pair $(A, B)$ is controllable and the pair $(A, C)$ is observable.

Assumption 2: The information on the control signal $u(t)$ on the time interval $[t-\bar{h}, t)$ can be stored and used for control design proposes.

The main goal of this paper is to present a control algorithm, which

- stabilizes the states of the system (1) at the origin;

- minimizes (in some sense) negative effects of exogenous disturbances, measurement noises and uncertainties of time delay.

\section{Attractive Ellipsoids Method for Time Delay SYSTEMS}

The classical optimal control concept addresses the problem of the minimization of some functional (for example, quadratic) subject to all possible trajectories of the system with admissible controls. Taking system uncertainties and disturbances into account requires robustification of the optimal control schemes. The optimality concept presented in the paper [24] considers the stabilization problem for the linear disturbed control system. It introduces a criterion based on the minimal attractive ellipsoid of the system, which characterizes an influence of disturbances to the closed-loop system. The optimal AEM control minimizes (in some sense) the disturbances effects (i.e. suppresses them). This concept is definitely very close to $H^{\infty}$ - control approaches. The similar control ideas were presented in the paper [26] in the beginning of 1980s. However, in that time the authors were not able to propose the effective computational schemes for control parameters tuning. Today control design using attractive ellipsoids methods admits LMI formalization [27], [24] and the scheme for optimal adjustment of control parameters can be represented as Semi-Definite Programming Problem [24], [23], [28].

In order to describe the principles of AEM for time-delay systems let us consider initially the control system with constant and known input delay:

$$
\dot{x}(t)=A x(t)+B u(t-h)+D f(t)
$$

where $x \in \mathbb{R}^{n}$ is the system state, the matrices $A, B, D$ are the same as in the equation (1), $h \in \mathbb{R}^{+}$is a given constant, disturbance function $f(t)$ satisfies (3) and $u \in \mathbb{R}^{n}$ is a fullstate predictor-based linear control [6]:

$$
u(t)=K z(t)
$$

where $L \in \mathbb{R}^{m \times n}$ is the matrix of the feedback gains and

$$
z(t)=e^{A h} x(t)+\int_{-h}^{0} e^{-A \theta} B u(t+\theta) d \theta .
$$

The predictor variable $z(t)$ satisfies the following delay-free differential equation

$$
\dot{z}(t)=(A+B K) z(t)+e^{A h} f(t) .
$$

Definition 3 ([24]): The ellipsoid

$$
\xi(P)=\left\{z \in \mathbb{R}^{n}: z^{T} P^{-1} z \leq 1\right\}, \quad P>0
$$

with the center in the origin and the configuration matrix $P$ is said to be invariant (attractive) for the system (9), (3) if the property

$$
\begin{gathered}
z(0) \in \xi(P) \text { implies } z(t) \in \xi(P) \text { for all } t>0, \\
(z(t) \rightarrow \xi(P) \text { as } t \rightarrow \infty)
\end{gathered}
$$

holds for any $f$ satisfying (3).

The attractive ellipsoids can be considered as a characteristic of the influence of the disturbances (3) to the system (6) in a steady mode. So, if the control $u$ provides minimal (in some sense) attractive ellipsoid to the closed-loop system (6), then it can be considered as an AEM optimal stabilizing controller, which minimizes the effects of the system disturbances [24].

The usual criterion of the ellipsoid minimality [24], [23], [28] is

$$
\text { minimize } \operatorname{trace}(P) \text {. }
$$

The trace of the matrix $P$ describes the sum of the squares of the ellipsoid's semiaxes.

For linear delay-free system (9) any attractive ellipsoid is also invariant [24]. However, for the original time-delay system (6) the attractive and invariant set may not coincide [12]. Indeed, let $\xi\left(P_{z}\right)$ be some invariant ellipsoid for the system (9). Then using the formula for the solution of the system (6) we obtain

$$
x(t+h)=e^{A h} x(t)+\int_{t}^{t+h} e^{(t+h-s) A} B u(s-h) d s+
$$




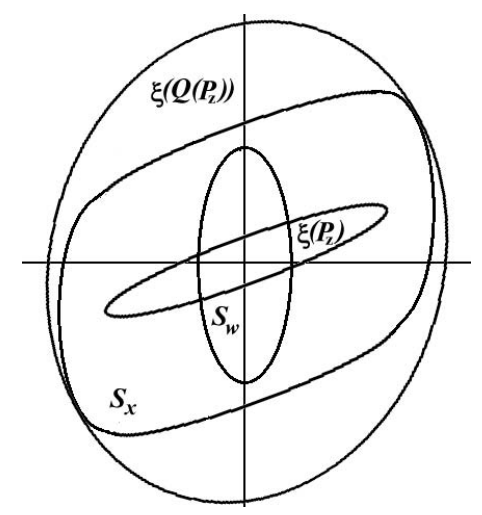

Fig. 1. The attractive set $S_{x}$ and its ellipsoidal estimate.

$$
\begin{gathered}
\int_{t}^{t+h} e^{(t+h-s) A} D f(s) d s=e^{A h} x(t)+ \\
\int_{-h}^{0} e^{-\theta A} B u(t+\theta) d \theta+\int_{0}^{h} e^{(h-\theta) A} D f(t+\theta) d \theta= \\
z(t)+\int_{0}^{h} e^{(h-\theta) A} D f(t+\theta) d \theta .
\end{gathered}
$$

The last integral term is called unavoidable stabilization error [12].

Let us denote by $S_{w}$ the set of all possible values of the unavoidable stabilization error, which is bounded due to (3). Then the obtained formula gives the attractive set $S_{x}=S_{x}\left(P_{z}\right)$ for the original system (6) (see, Fig. 1 ) in the form

$$
S_{x}=\xi\left(P_{z}\right) \dot{+} S_{w},
$$

where $\xi\left(P_{z}\right)$ is an invariant ellipsoid for the prediction system (9) and the symbol $\dot{+}$ means the Minkowski (geometrical) sum of the sets (see, for example, [29]).

It is easy to see, that $y(t) \rightarrow \xi\left(P_{z}\right)$ implies $x(t) \rightarrow S_{x}$, so $S_{x}$ is attractive set. However, in the general case $x(0) \in S_{x}$ does not imply $z(0) \in \xi\left(P_{z}\right)$, so $S_{x}$ may not be invariant.

The uncertain function $f(t)$ is bounded for each time moment and belongs to ellipsoid $\xi\left(Q_{f}^{-1}\right)$. Regardless of the fact that for any $t$ and $\theta$ the value $e^{(h-\theta) A} D f(t+\theta)$ also belongs to the some ellipsoid, the sets $S_{w}$ and $S_{x}$ may not be elliptic. Due to this in [12] it was introduced the optimization criterion for the attractive set $S_{x}\left(P_{z}\right)$ based on ellipsoidal estimate $\xi\left(Q\left(P_{z}\right)\right)$ of this set (see, Fig. 1), where $Q\left(P_{z}\right)$ is a configuration matrix of minimal (with respect to trace criteria) ellipsoid, which estimates of the set $S_{x}\left(P_{z}\right)$. Due to linearity of the criterion (11) the proposed construction of attractive ellipsoid reduces the problem of the AEM optimal control design for the original system (6) to the same problem for the predictor system (9) (see, Theorem 1 from [12] for the details). The present paper uses this result as motivation for applying attractive ellipsoids technique only to the predictor system even when an input delay is unknown and time-varying.

\section{Stabilizing CONTROL DESIGN}

\section{A. Predictor-based output feedback}

Introduce a Luenberger-like observer of the form

$$
\dot{\hat{x}}=(A+\mathcal{L} C) \hat{x}+B u(t-\underline{h})-\mathcal{L} y(t),
$$

where the matrix $\mathcal{L} \in \mathbb{R}^{n \times k}$ is needed to be defined.

Consider also the error equation

$$
\dot{e}=(A+\mathcal{L} C) e+B(u(t-\underline{h})-u(t-h(t)))-D f-\mathcal{L} E g,
$$

where $e=\hat{x}-x$.

Select the control in the form

$$
u(t)=\mathcal{K} z(t)
$$

where the matrix of control $\mathcal{K} \in \mathbb{R}^{m \times n}$ is needed to be designed and

$$
z(t)=e^{A \underline{h}} \hat{x}(t)+\int_{-\underline{h}}^{0} e^{-A \theta} B u(t+\theta) d \theta
$$

is the predictor variable (estimating the state $\hat{x}$ at time $t+\underline{h}$ ).

In this case the closed-loop system can be rewritten in the form

$$
\left\{\begin{array}{l}
\dot{e}=(A+\mathcal{L} C) e+B \mathcal{K} \int_{t-h(t)}^{t-\underline{h}} \dot{z}(\tau) d \tau-D f-\mathcal{L} E g, \\
\dot{z}=(A+B \mathcal{K}) z+e^{A \underline{h}} \mathcal{L} C e .
\end{array}\right.
$$

Theorem 4: If the tuple $\left(\alpha, \tau_{1}, \tau_{2}, \mathcal{X}, \mathcal{Z}, \mathcal{L}, \mathcal{Y}\right)$ satisfies the bilinear matrix inequality

$$
\begin{gathered}
\left(\begin{array}{cccccc}
\Pi_{1} & \Pi_{21}^{T} & \Pi_{21}^{T} & B \mathcal{Y} & D & \mathcal{L} E \\
\Pi_{21} & \Pi_{2} & \Pi_{23} & 0 & 0 & 0 \\
\Pi_{21} & \Pi_{23}^{T} & \Pi_{3} & 0 & 0 & 0 \\
\mathcal{Y}^{T} B^{T} & 0 & 0 & -\frac{1}{e^{\alpha h}} \mathcal{R} & 0 & 0 \\
D^{T} & 0 & 0 & 0 & -\tau_{1} Q_{f} & 0 \\
E^{T} \mathcal{L}^{T} & 0 & 0 & 0 & 0 & -\tau_{2} Q_{f}
\end{array}\right) \leq 0 \\
\Pi_{1}=A \mathcal{Z}+\mathcal{Z} A^{T}+\mathcal{L} C \mathcal{Z}+\mathcal{Z} C^{T} \mathcal{L}^{T}+\alpha \mathcal{Z}, \quad \mathcal{Z}>0 \\
\Pi_{2}=A \mathcal{X}+\mathcal{X} A^{T}+B \mathcal{Y}+\mathcal{Y}^{T} B^{T}+\alpha \mathcal{X}, \quad \mathcal{X}>0 \\
\Pi_{21}=e^{A h} \mathcal{L} C \mathcal{Z}, \Pi_{23}=A \mathcal{X}+B \mathcal{Y}, \Pi_{3}=\mathcal{R}-\frac{1}{\Delta h} \mathcal{X} \\
\mathcal{Z}, \mathcal{X}, \mathcal{R} \in \mathbb{R}^{n \times n}, \mathcal{Y} \in \mathbb{R}^{m \times n}, \mathcal{L} \in \mathbb{R}^{n \times k}, \mathcal{R}>0
\end{gathered}
$$

then

$$
\varepsilon(\mathcal{Z}, \mathcal{X}):=\left\{e \in \mathbb{R}^{n}, z \in \mathbb{R}^{n}: e^{T} \mathcal{Z}^{-1} e+z^{T} \mathcal{X}^{-1} z \leq 1\right\}
$$

is exponentially attractive ellipsoid of the system (16) with $\mathcal{K}=\mathcal{Y} \mathcal{X}^{-1}$.

The proof of this theorem is based on LyapunovKrasovskii functional method. It considers the functional of 
the form

$$
\begin{gathered}
V(t, e(t), z(t), \dot{z}(\cdot))= \\
e^{T}(t) \mathcal{Z}^{-1} e(t)+z^{T}(t) \mathcal{X}^{-1} z(t)+V_{1}(t, \dot{z}(\cdot))+V_{2}(t, \dot{z}(\cdot)), \\
V_{1}(t, \dot{z}(\cdot))=(\Delta h)^{2} \int_{t-\underline{h}}^{t} e^{\alpha(s-t+\underline{h})} \dot{z}^{T}(s) \tilde{\mathcal{R}} \dot{z}(s) d s \\
V_{2}(t, \dot{z}(\cdot))=\Delta h \int_{-\Delta h t-\underline{h}+\theta}^{0} \int_{t-\underline{h}}^{t-} e^{\alpha(s-t+\underline{h})} \dot{z}^{T}(s) \tilde{\mathcal{R}} \dot{z}(s) d s d \theta,
\end{gathered}
$$

where $\tilde{\mathcal{R}}=e^{-\gamma \underline{h}} \mathcal{X}^{-1} \mathcal{R} \mathcal{X}^{-1}$.

The next lemma treats the question about feasibility of the system of matrix inequalities (17)-(21) and other relaxations to SDP tools will also be given later on.

Lemma 5: Under Assumption 1 the system of matrix inequalities (17)-(21) is feasible at least for small $\Delta h$.

This lemma can be proven in two steps. Firstly, we consider the reduced system of matrix inequalities, which is obtained from (17)-(21) for $\Delta h \rightarrow 0$. Secondly, we use the properties of controllability and observability of the pairs $\{A, B\},\{A, C\}$ in order to see feasibility of the reduced system of matrix inequalities.

\section{B. Adjustment of Control Parameters: Computational As- pects}

The predictor variable $z$ estimates the future state of observer variable $\hat{x}(t+\underline{h})$ and the vector $e$ describes the observation error $e=\hat{x}-x$, so in order to improve control precision we may minimize the size of the attractive ellipsoid of the system (16), i.e. we need to solve the following optimization problem

$$
\begin{gathered}
\operatorname{minimize} \text { trace } \mathcal{Z}+\text { trace } \mathcal{X} \\
\text { s.t. }(17)-(21) .
\end{gathered}
$$

The optimization (23), (17)-(21) has the linear cost functional and the constraints represented in the form of Bilinear Matrix Inequality (BMI). For low order systems, such a problem can be solved by some BMI solver, for example, PENBMI. In the same time, the constraints can be restricted in order to obtain SDP problem.

Lemma 6: If for some $\alpha, \beta \in \mathbb{R}_{+}$the following LMI system is feasible:

$$
\begin{gathered}
\left(\begin{array}{cc}
-\mathcal{S} & {\left[\begin{array}{c}
\mathcal{F} C \\
\mathcal{F} C
\end{array}\right]^{T}} \\
{\left[\begin{array}{c}
\mathcal{F} C \\
\mathcal{F} C
\end{array}\right]} & -\mathcal{V}
\end{array}\right) \leq 0, \mathcal{S}>0, \mathcal{V}>0 \\
\left(\begin{array}{cc}
-\frac{\beta}{e^{\alpha h}} I_{n} & B \mathcal{Y} \\
\mathcal{Y}^{T} B^{T} & -\mathcal{R}
\end{array}\right) \leq 0
\end{gathered}
$$

$$
\left(\begin{array}{cccc}
\mathcal{Q} A+A^{T} \mathcal{Q}+ & & & \\
\mathcal{F} C+C^{T} \mathcal{F}+ & \mathcal{Q} & \mathcal{Q} D & \mathcal{F} E \\
\alpha \mathcal{Q}+S & & & \\
\mathcal{Q} & -\frac{1}{\beta} I_{n} & 0 & 0 \\
D^{T} Q & 0 & -\tau_{1} Q_{f} & 0 \\
E^{T} \mathcal{F}^{T} & 0 & 0 & -\tau_{2} Q_{g}
\end{array}\right) \leq 0
$$

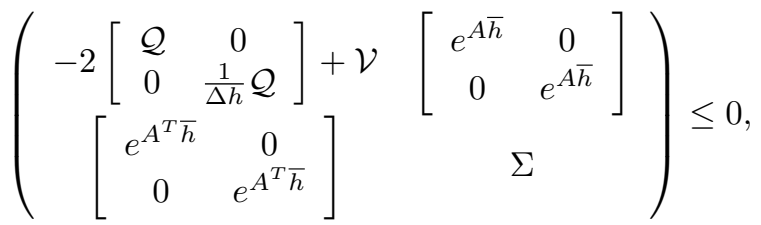

$$
\begin{aligned}
& \alpha \geq \tau_{1}+\tau_{2}, \mathcal{R}>0, \mathcal{Q}>0, \mathcal{X}>0, \mathcal{V}>0, \\
& \Sigma:=\left[\begin{array}{cc}
\Pi_{2} & \sqrt{\Delta h}(A \mathcal{X}+B \mathcal{Y}) \\
\sqrt{\Delta h}(A \mathcal{X}+B \mathcal{Y})^{T} & \Delta h \mathcal{R}-\mathcal{X}
\end{array}\right], \\
& \mathcal{Q}, \mathcal{X}, \mathcal{S} \in \mathbb{R}^{n \times n}, \mathcal{Y} \in \mathbb{R}^{m \times n}, \mathcal{F} \in \mathbb{R}^{n \times k}, \mathcal{V} \in \mathbb{R}^{2 n \times 2 n}
\end{aligned}
$$

then the tuple $(\alpha, \mathcal{X}, \mathcal{Z}, \mathcal{L}, \mathcal{Y})$, where $\mathcal{X}=\mathcal{Q}^{-1}$ and $\mathcal{L}=$ $\mathcal{Q}^{-1} \mathcal{F}$, satisfies (17)-(21).

This lemma allows to organize the procedure for controller and observer parameters tuning basing on semidefinite programming technique. Indeed, for any fixed $\alpha, \beta \in \mathbb{R}^{+}$the system of matrix inequalities (24)-(28) becomes LMI form. So, in order to minimize the attractive ellipsoid (22) we need to solve the following optimization problem

$$
\begin{gathered}
\operatorname{minimize} \operatorname{trace}\left(\mathcal{Q}^{-1}\right)+\operatorname{trace} \mathcal{X} \\
\text { s.t. }(24)-(28) .
\end{gathered}
$$

The cost functional of the problem is nonlinear now. Fortunately, this optimization problem is equivalent the following SDP problem

$$
\begin{gathered}
\operatorname{minimize} \operatorname{trace}(\mathcal{H})+\operatorname{trace}(\mathcal{X}) \\
\text { s.t. }(24)-(28) \text { and } \\
\left(\begin{array}{cc}
\mathcal{H} & I_{n} \\
I_{n} & \mathcal{Q}
\end{array}\right) \geq 0, \mathcal{H}>0, \mathcal{H} \in \mathbb{R}^{n \times n} .
\end{gathered}
$$

This equivalence obviously follows from the inequality $\mathcal{H} \geq$ $\mathcal{Q}^{-1}$ and Shur complement.

For a given $\alpha, \beta \in \mathbb{R}_{+}$let us denote by $J(\alpha, \beta)$ the solution of the optimization problem (29). The corresponding solution can be found using any SDP solver (for example, SeDuMi). In order to minimize the function $J(\alpha, \beta)$ some derivative-free method can be used (for example, the procedure fminsearch of MATLAB).

Lemma 6 does not prove equivalence between conditions (17)-(21) and (24)-(28), since its proof is based on $\Lambda$ inequality (Young's matrix inequality) [30]. Therefore, the presented optimization scheme may give only suboptimal and a rather conservative solution, which can be considered just as an approximation for the AEM optimal feedback.

\section{NUMERICAL EXAMPLE}

Consider the system (1) with parameters

$$
\begin{gathered}
A=\left(\begin{array}{ccc}
-0.1 & 1 & 0 \\
-0.1 & 0 & -0.4 \\
0 & -0.1 & 0.2
\end{array}\right), B=\left(\begin{array}{l}
1 \\
0 \\
1
\end{array}\right), \\
C=\left(\begin{array}{lll}
1 & 0 & 0 \\
0 & 0 & 1
\end{array}\right), D=\left(\begin{array}{c}
1 \\
0.5 \\
0
\end{array}\right), E=\left(\begin{array}{ll}
1 & 0 \\
0 & 1
\end{array}\right), \\
Q_{f}=10^{4} \text { and } Q_{g}=10^{4}\left(\begin{array}{cc}
2 & -0.3 \\
-0.3 & 1.5
\end{array}\right),
\end{gathered}
$$




$$
\underline{h}=0.35, \quad \bar{h}=0.5 \text {. }
$$

The matrix $A$ is unstable, $\lambda_{1}=0.273, \lambda_{2,3}=-0.0869 \pm$ $0.2830 i$. The selected matrices $Q_{f}$ and $Q_{g}$ correspond to disturbances and noises of the order $\mathcal{O}\left(10^{-2}\right)$.

Using the optimization procedure (29) for $\alpha=0.05$ and $\beta=0.004$ we obtain the following suboptimal solution:

$$
\begin{gathered}
K_{\text {subopt }}=\left(\begin{array}{ccc}
-0.0232 & 0.0943 & -0.4889
\end{array}\right), \\
L_{\text {subopt }}=\left(\begin{array}{cc}
-1.4276 & -0.4486 \\
-0.5563 & 0.1173 \\
0.3131 & -0.6700
\end{array}\right) .
\end{gathered}
$$

The numerical simulation results for the obtained output feedback control application are depicted on Fig. 2-5. They have been done for

$$
\begin{gathered}
h(t)=0.35+0.15 \sin ^{2}(t), \\
f(t, x)=0.01 \cos (t), \\
g(t)=\left(\begin{array}{c}
0.0036 \sin (3 t)-0.0062 \cos (3 t) \\
0.0078 \sin (3 t)+0.0029 \cos (3 t)
\end{array}\right), \\
x_{0}=(0.5,0,-0.1)^{T} \text { and } u(t)=0 \text { for } t \in[-\bar{h}, 0] .
\end{gathered}
$$

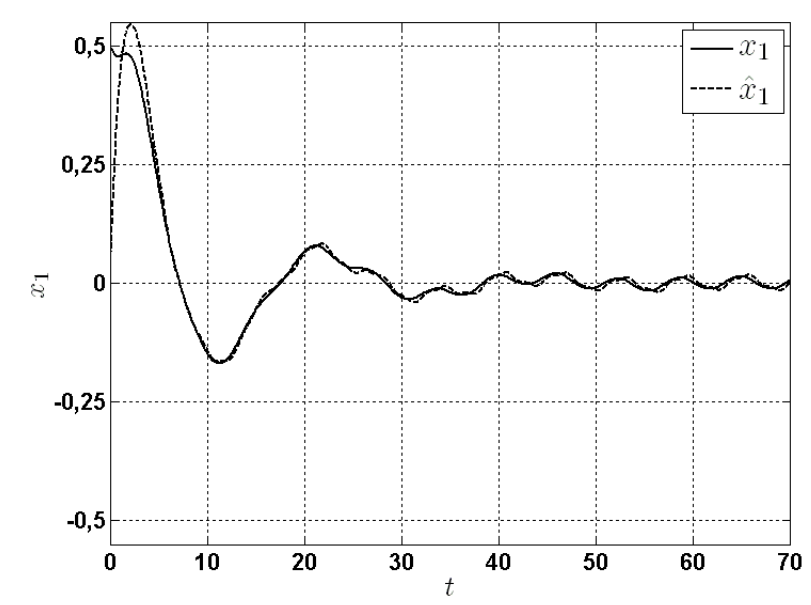

Fig. 2. Evolution of real and observed state $x_{1}$.

\section{CONCLUSIONS}

The paper presents the output-based robust control for linear time-invariant system with unknown bounded input delay. The design procedure combines Luenberger observer with predictor scheme. Stability analysis of the closed-loop system is based on the method of Lyapunov-Krassovski functionals. Robustness properties of the presented control scheme is studied using attractive ellipsoid method. Procedure of control parameters tuning for optimal reduction of exogenous disturbances and measurement noises is formulated as an optimization problem with linear cost functional and constraints presented in the form of bilinear matrix inequalities. The scheme for finding a suboptimal solution of the obtained bilinear problem is presented using semidefinite programming technique. It is also important to study the

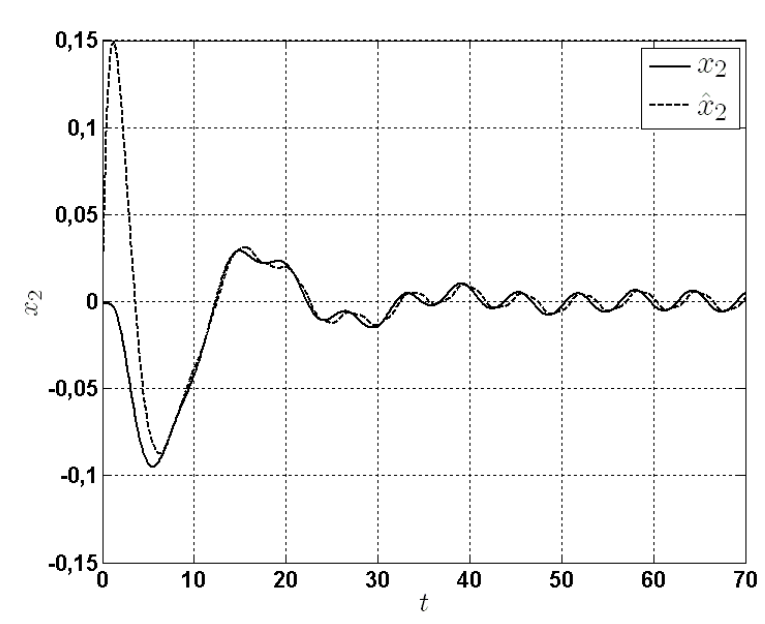

Fig. 3. Evolution of real and observed state $x_{2}$.

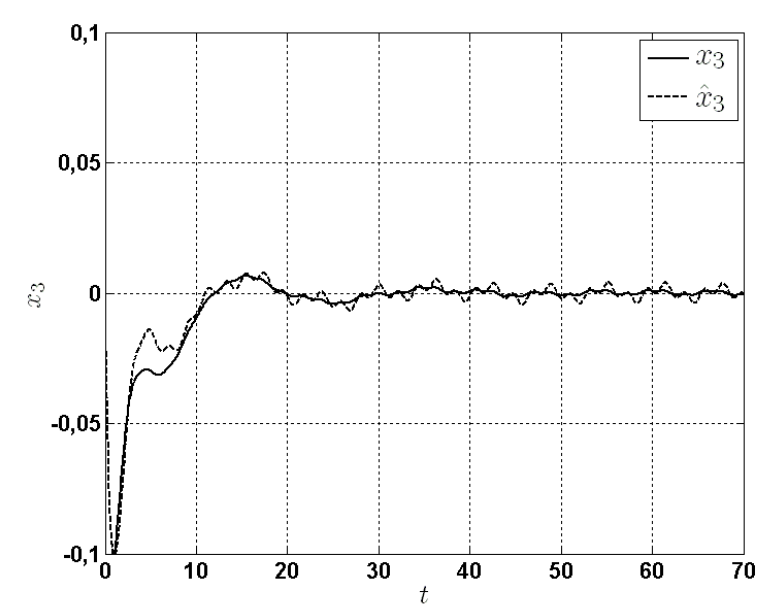

Fig. 4. Evolution of real and observed state $x_{3}$.

robustness of the method with respect to parametric uncertainties, for example, in matrices $A, B, C$. This problem is opened for future research.

\section{REFERENCES}

[1] A. Kruszewski, W. J. Jiang, E. Fridman, J.-P. Richard, and A. Toguyeni, "A switched system approach to exponential stabilization through communication network," IEEE Transactions on Control Systems Technology, vol. 20(4), pp. 887-900, 2012.

[2] G. Stephanopoulos, Chemical process control: an introduction to theory and practice. Prentice-Hall, 1984.

[3] A. C. Flower and M. C. Mackey, "Relaxation oscillations in a class of delay differential equations," SIAM Journal of Applied Mathematics, vol. 63, pp. 299-323, 2002.

[4] S.-B. Choi and J. Hedrick, "Robust throttle control of automotive engines," ASME Journal of Dynamic Systems, Measurement and Control, vol. 118, pp. 92-98, 1996.

[5] M. Yefremov, A. Polyakov, and V. Strygin, "An active stabilization algorithm for a spacecraft with viscoelastic elements under uncertainties," Journal of Applied Mathematics and Mechanics, vol. 70(5), pp. 800-811, 2006.

[6] Z. Artstein, "Linear systems with delayed controls: A reduction," IEEE Transactions on Automatic Control, vol. 27(4), pp. 869-879, 1982. 


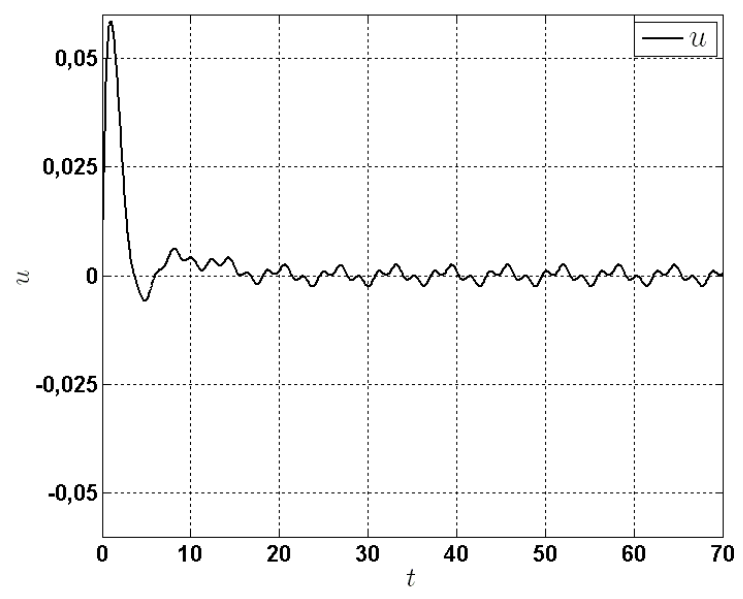

Fig. 5. Control signal $u$.

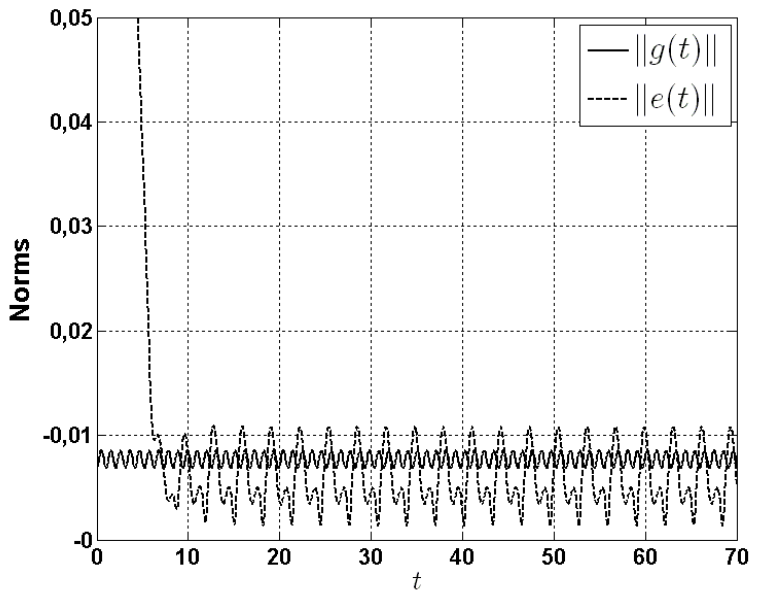

Fig. 6. The norms of the vectors of measurement noises $g(t)$ and observation errors $e(t)$.

[7] K. Watanabe, "Finite spectrum assignment and observer for multivariable systems with commensurate delays," IEEE Transactions on Automatic Control, vol. 31(6), pp. 543-550, 1986.

[8] J.-P. Richard, "Time-delay systems: An overview of some recent advances and open problems," Automatica, vol. 39(10), pp. 16671694, 2003.

[9] M. Krstic, Delay Compensation for Nonlinear, Adaptive, and PDE Systems. Birkhauser Boston, 2009.

[10] N. Bekiaris-Liberis and M. Krstic, "Compensation of time-varying input and state delays for nonlinear systems," Journal of Dynamic Systems, Measurement, and Control, vol. 134(1), p. paper 011009, 2012.

[11] F. Mazenc, S. Niculescu, and M. Krstic, "Lyapunov-Krasovskii functionals and application to input delay compensation for linear timeinvariant systems," Automatica, vol. 48(7), pp. 1317-1323, 2012.

[12] A. Polyakov, "Minimization of disturbances effects in time delay predictor-based sliding mode control systems," Journal of The Franklin Institute, vol. 349(4), pp. 1380-1396, 2012.

[13] E. Fridman, A. Seuret, and J.-P. Richard, "Robust sampled-data stabilization of linear systems: an input delay approach," Automatica, vol. 40(8), pp. 1441-1446, 2004.

[14] A. Polyakov, "On practical stabilization of the systems with relay delay control," Automation and Remote Control, vol. 71(11), pp. 2331-2344, 2010.

[15] X. Han, E. Fridman, and S. Spurgeon, "Sliding mode control in the presence of input delay: A singular perturbation approach," Automatica, vol. 48(8), pp. 1904-1912, 2012.

[16] M. Dambrine, F. Gouaisbaut, W. Perruquetti, and J.-P. Richard, "Robustness of sliding mode control under delays effects : a case study," in 2nd IEEE-IMACS Conference CESA98, 1998, pp. 817-821.

[17] H.-L. Choi and J.-T. Lim, "Output feedback regulation of a chain of integrators with an unknown time-varying delay in the input," IEEE Transactions on Automatic Control, vol. 55(1), pp. 263-268, 2010.

[18] A. Polyakov, D. Efimov, W. Perruquetti, and J.-P. Richard, "Interval observer approach to output stabilization of time-varying input delay system," in European Control Conference, 2013, pp. 4412-4417.

[19] _ _ "Output stabilization of time-varying input delay systems using interval observation technique," Automatica, 2014, (accepted).

[20] A. Seuret, T. Floquet, J.-P. Richard, and S. K. Spurgeon, "A sliding mode observer for linear systems with unknown time varying delay," in American Control Conference, 2007, pp. 4558-4563.

[21] G. Zheng, J.-P. Barbot, D. Boutat, T. Floquet, and J.-P. Richard, "On obserability of nonlinear time-delay systems with unknown inputs," IEEE Transactions on Automatic Control, vol. 56(8), pp. 973-1978, 2011.

[22] B. Polyak and M. Topunov, "Suppression of bounded exogenous disturbances: Output feedback," Automation and Remote Control, vol. 69, pp. 801-818, 2008.

[23] S. Gonzalez-Garcia, A. Polyakov, and A. Poznyak, "Using the method of invariant ellipsoids for linear robust output stabilization of spacecraft," Automation and Remote Control, vol. 72(3), pp. 540-555, 2011.

[24] S. Nazin, B. Polyak, and M. Topunov, "Rejection of bounded exogenous disturbances by the method of invariant ellipsoids," Automation and Remote Control, vol. 68, pp. 467-486, 2007.

[25] S. Gonzalez-Garcia, A. Polyakov, and A. Poznyak, "Output linear feedback for a class of nonlinear systems based on the invariant ellipsoid method," in 5th International Conference on Electrical Engineering, Computing Science and Automatic Control, Mexico-City, 2008, pp. 7-12.

[26] P. Usoro, F. Schweppe, L. Gould, and D. Wormley, "A Lagrange approach to set-theoretic control synthesis," IEEE Transactions on Automatic Control, vol. 27(2), pp. 393-399, 1982.

[27] S. Boyd, E. Ghaoui, E. Feron, and V. Balakrishnan, Linear Matrix Inequalities in System and Control Theory. Philadelphia: SIAM, 1994.

[28] A. Polyakov and A. Poznyak, "Invariant ellipsoid method for minimization of unmatched disturbances effects in sliding mode control," Automatica, vol. 47(7), pp. 1450-1454, 2011.

[29] A. Kurzhanski and I. Valyi, Ellipsoidal Calculus for Estimation and Control. Boston, MA: Birkhauser, 1997.

[30] A. Poznyak, Advanced Mathematical Tools for Automatic Control Engineers: Deterministic Techniques. Elsevier, 2008. 